\title{
Mathematically modeling fluid flow and fluid shear stress in the canaliculi of a loaded osteon
}

\author{
Xiaogang Wu, Ningning Wang, Zhaowei Wang, Weilun Yu, Yanqin Wang, Yuan Guo and Weiyi Chen*
}

\author{
*Correspondence: \\ chenweiyi211@163.com \\ Shanxi Key Lab. of Material \\ Strength \& Structural \\ Impact and College \\ of Mechanics, Taiyuan \\ University of Technology, \\ Taiyuan 030024, People's \\ Republic of China
}

\begin{abstract}
Background: Mechanical load-induced intraosseous pressure gradients may result in some fluid stimuli effects, such as fluid flow and fluid shear stress (FSS), which may enable bone cells to detect external mechanical signals. Interstitial bone fluid flow is known to occur in lacunar-canalicular porosity (PLC).

Methods: In order to characterize lacunar-canalicular fluid flow behavior, a hierarchical osteon system is developed. The osteon is modeled as a poroelastic annular cylinder with two types of impermeable boundary cases considered on its outer wall: one is elastic restrained (Case I), whereas the other is displacement confined (Case II). Analytical solutions such as canalicular fluid velocity, pressure, fluid flow rate (FFR), and shear stress are obtained.

Results: Results show that the amplitudes of FFR and FSS are proportional to strain amplitude and frequency. However, the key loading factor governing canalicular fluid flow behavior is the strain rate. The larger canalicular radius is, the larger amplitudes of FFR and FSS generalized, especially, the FSS amplitude is proportional to canalicular radius. In addition, both FFR and FSS amplitudes produced in case II are larger than those of case I.
\end{abstract}

Conclusion: Strain rate can be acted as a representative loading parameter governing the canalicular fluid flow behavior under a physiological state. This model can facilitate better understanding the load induced the fluid permeation in the PLC. The approach can also be used to analyze the structure of the proteoglycan matrix in the fluid space surrounding the osteocytic process in the canaliculus.

Keywords: Canaliculi, Fluid flow rates (FFR), Fluid shear stress (FSS), Poroelasticity, Osteon

\section{Background}

As a poroelastic material, bone often bears cyclic loads that might come from walking, running, or other daily activities [1]. These activities enable bones to "feel" and "adapt", this is the mechanism called bone mechanotransduction. Intraosseous pressure gradients may result in some fluid stimuli effects (fluid flow, fluid shear stress (FSS), and streaming potentials), which may enable bone cells to initiate the process of adapting bone mass and structure to the environment [2]. This process may be the basic mechanism of bone mechanotransduction. Although the constituents and ultrastructure of

( ) The Author(s) 2016. This article is distributed under the terms of the Creative Commons Attribution 4.0 International License (http://creativecommons.org/licenses/by/4.0/), which permits unrestricted use, distribution, and reproduction in any medium, provided you give appropriate credit to the original author(s) and the source, provide a link to the Creative Commons license, and indicate if changes were made. The Creative Commons Public Domain Dedication waiver (http://creativecommons.org/publicdomain/zero/1.0/) applies to the data made available in this article, unless otherwise stated. 
bone interstitial fluid pathways remain poorly understood because of technical difficulties, interstitial fluid flow in bone tissue has been suggested to serve an important role in bone adaptation and metabolism [2].

Research for the intraosseous fluid stimuli should be conducted at the osteon level [1]. Osteon is the basic bone structure unit with a cylindrical structure, which is made of lamella around a Haversian canal. The Haversian canal contains blood vessel(s), nerve, and some spaces occupied by bone fluid. The wall of a Haversian canal is covered with cells, and behind the cells is the entrance to the bone canaliculi [3] - the passageways between lacunae and the Haversian canal. The space in the lacunae and the canaliculi is called lacunar-canalicular porosity (PLC). Mechanical load induced interstitial bone fluid flow is known to occur in this porosity [3-6]. This porosity also provides an ideal milieu for transfer of exogenous and endogenous signals via mechanical, electrical, and chemical mechanisms [7].

The PLC dimension is characterized by the radius of the canaliculus (order $=0.1 \mu \mathrm{m}$ ) [3]. The canaliculi have been regarded as the passageway that allows nutrients, oxygen, and wastes to be exchanged between blood vessels within the Haversian canal and osteocytes [8]. The canaliculus is the canal associated with slower relaxation of excess pore pressure and may also serve an important mechanosensory role [8] by providing the channels through which osteocytes can sense FSS $[3,4]$.

FSS plays an important role in modulating cell functions, and several laboratory systems have applied FSS to cells [9]. Theoretical models have been established to investigate intraosseous fluid flow behavior and FSS in homogeneous porous bone specimens $[6,10,11]$ or in single osteon [12-17]. However, the osteon has different properties in longitudinal and radial directions, and the fluid flow in canaliculi is not directly coupled with mechanical loading [1]. Thus, a transverse isotropic poroelastic hollow osteon model [18-23] is developed to simulate osteonal poroelastic behavior. However, these theoretical models do not directly link the mechanical loading at the osteon scale to the scale of canalicular fluid flow.

Many theoretical models $[6,16,24-26]$ have been established to deduce the canalicular fluid flow behavior, but its mechanism remains poorly understood because it is difficult for obtaining reliable experimental information in vivo at the nanoscale. Some authors have identified the canalicular fluid flow evaluated from Darcy's law or Brinkman's equation [6,27]. To amend the above zero charge flux assumption, a highlighted model (by using the linearity of the Stokes-Brinkman differential equation) on the basis of an up-scaling approach has been proposed to quantify electroosmotic and hydro electrochemical effects [28-31]. Canalicular fluid flow associates with hydraulic, chemical, and electrical phenomena. Hydraulic flow and shear stress are generally the most relevant fluid stimuli effects induced by pressure differences in the interstitial bone fluid [32]. The aforementioned models are complicated and expatiatory, but those physical effects are not truly coupling in the motion equation of solutions (e.g., in Sansalone et al. [32] "Hydraulic, osmotic, and electroosmotic contributions to the fluid transport were split and treated independently using the linearity of the Stokes-Brinkman differential equation"). In other words, fluid motion equations might no longer work for those coupled physical effects. Chemical, osmotic, and electroosmotic phenomena are hysteresis band effects induced by canalicular fluid flow. The first physical effects of canalicular fluid flow behavior are fluid pressure gradient, velocity, and viscous force. 
Therefore, we will establish a hierarchical model to directly evaluate the first physical effects of canalicular fluid transport behavior, which allows the linking of cyclic mechanical loading on osteon to the fluid flow in the canaliculi. The lacunar-canalicular primary porosity scale is proposed to relax the pore pressure associated with FSS because of mechanical loading. A homogenization canaliculated porosity approach may avoid multiscale parameters of permeability. We specifically develop a canaliculi model on the basis of our previous osteon model [33-36] to link the external loads to the canalicular fluid velocity, pressure, flow rates, and shear stress. The model can also be used to analyze the structure of the proteoglycan matrix in the fluid space surrounding the osteocytic process in the canaliculus.

\section{Methods}

Biot's poroelastic theory is used to account for fluid-solid interactions in osteon tissue. According to $\mathrm{Wu}$ et al. [36], the osteon system is modeled as a hierarchical hollow annular cylinder structure (as shown in Fig. 1) and two types of boundary cases for the osteon wall are proposed (as shown in Fig. 2). In this hierarchical structure, the canaliculi are assumed to be straight tubes and evenly distributed in the osteon wall, which run across the lamella and stop at the cement line. The viscous canalicular fluid flow and shear

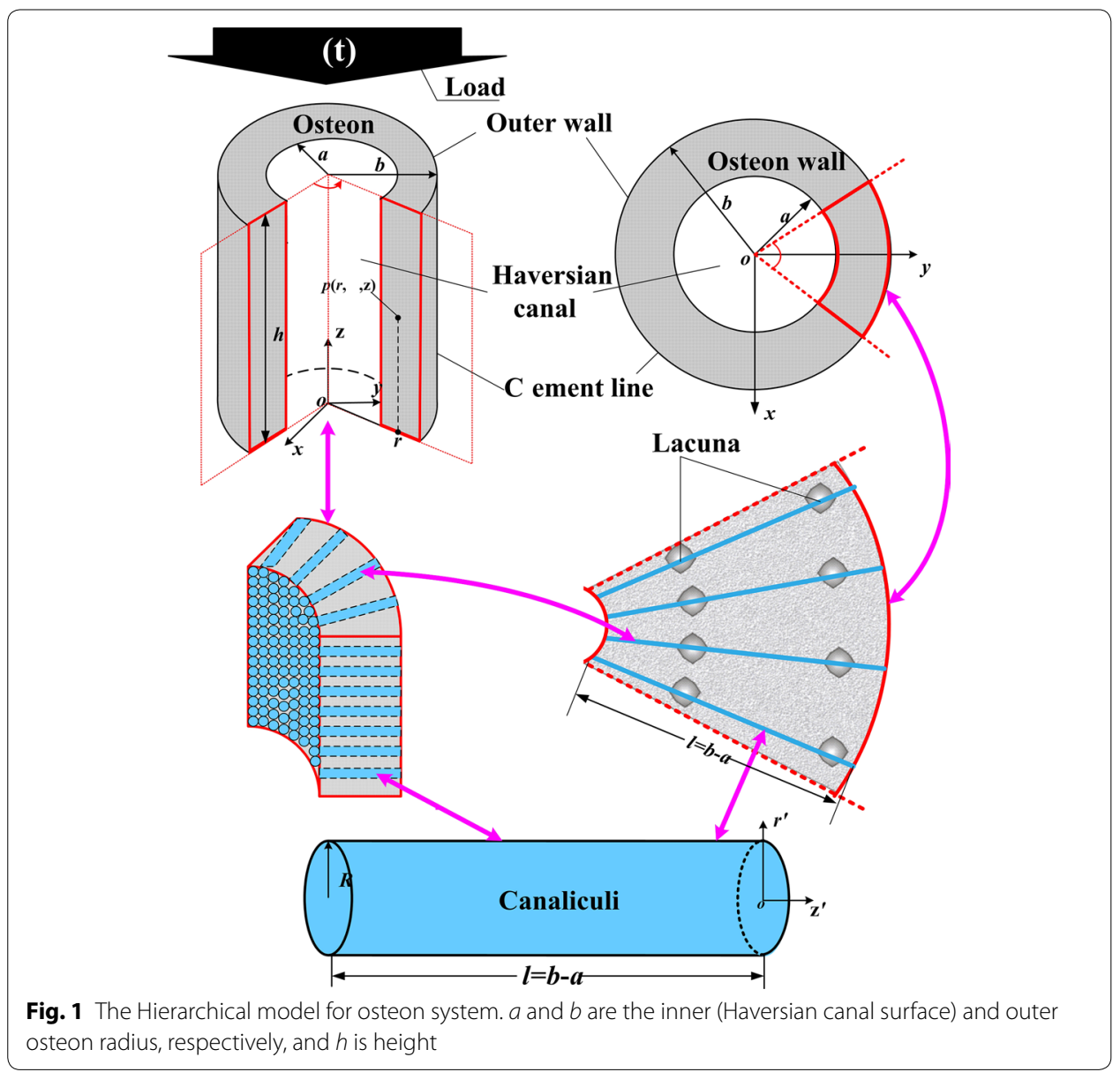




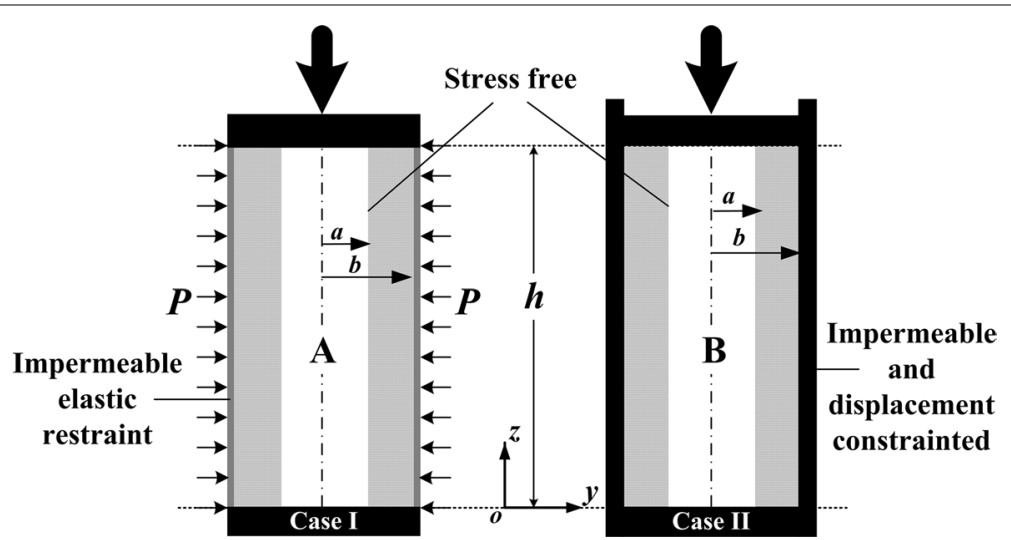

Fig. 2 The osteon outer wall in case I and case II are assumed to be elastic restrained ( $\mathbf{A})$ and displacement confined (B) respectively

stress on the canalicular wall are induced by the fluid pressure difference and the effect of lacuna is neglected.

According to $\mathrm{Wu}$ et al. [36], the solutions of whole pore pressure distribution $p_{m}=p_{0 m}(r) e^{i \omega t}(\omega$ is the angular frequency of loading, $m=1$ is for case I and $m=2$ is for case II) in the osteon wall is given by:

$$
p_{m}=\frac{M M_{11}\left(\alpha c_{m}+\alpha^{\prime} \varepsilon_{z 0}\right)}{M_{11}+M \alpha^{2}}\left[\frac{I_{0}(C r) K_{1}(C b)+K_{0}(C r) I_{1}(C b)}{I_{0}(C a) K_{1}(C b)+I_{1}(C b) K_{0}(C a)}-1\right] e^{i \omega t},
$$

where, $M_{11}=E_{r}\left(E_{z}-E_{r} \mu_{z}^{2}\right)\left(1+\mu_{r}\right)^{-1}\left(E_{z}-E_{z} \mu_{r}-2 E_{r} \mu_{z}^{2}\right)^{-1}$ is an elastic component of the stiffness tensor. $E_{r}$ and $E_{z}$ are radial and longitudinal Young modulus, $\mu_{r}$ and $\mu_{z}$ are radial and longitudinal drained Poisson's ratio, $I_{n}$ and $K_{n}$ are the first kind and the second kind modified Bessel function of order $n$ respectively, and $c_{m}$ is constant and can be found in Ref. [33]. The constant $\mathrm{C}$ is given by Wu et al. [1,33-36].

$$
C=\sqrt{i \omega \mu\left(M_{11}+M \alpha^{2}\right) /\left(k M M_{11}\right)} .
$$

where $i=\sqrt{-1}, \mu$ and $k$ are dynamic viscosity and intrinsic permeability, respectively.

According to the established canaliculi model in Wu et al. [36], the solutions for pressure $p^{\prime}$ and longitudinal velocity component $u$ in the canaliculi are presented as:

$$
\begin{aligned}
& p^{\prime}\left(z^{\prime}\right)=\left[p_{0}(a)+\frac{p_{0}(b)-p_{0}(a)}{l}\left(z^{\prime}-a\right)\right] e^{i \omega t}, \\
& u\left(r^{\prime}\right)=\frac{p_{0}(a)-p_{0}(b)}{i \omega \rho l}\left[1-\frac{I_{0}\left(\beta r^{\prime}\right)}{I_{0}(\beta R)}\right] e^{i \omega t} .
\end{aligned}
$$

where, $\rho$ is the fluid density, and $\mu$ is the dynamic viscosity. The canalicular radius is $R$ and lengthened $l(l=b-a)$. The constant $\beta$ is given by.

$$
\beta=\sqrt{i \omega / \mu}
$$


According to Weinbaum et al. [6] and You et al. [37], the fluid flow rate, $Q$, and shear stress, $\tau_{w}$, on the canalicular wall or osteocyte process membrane are given by:

$$
\begin{aligned}
& Q=\int_{0}^{R} 2 \pi r^{\prime} u\left(r^{\prime}\right) d r^{\prime}, \\
& \tau_{w}=\left.\mu \frac{\partial u\left(r^{\prime}\right)}{\partial r^{\prime}}\right|_{r^{\prime}=R} .
\end{aligned}
$$

Thus finally, we can obtain the FFR and FSS:

$$
\begin{aligned}
Q_{m} & =\frac{2 \pi}{i \omega \rho l} p_{0 m}(b)\left[\frac{R}{\beta} \frac{I_{1}(\beta R)}{I_{0}(\beta R)}-\frac{R^{2}}{2}\right] e^{i \omega t} \\
\tau_{w m} & =\frac{\mu \beta}{i \omega \rho l} p_{0 m}(b) \frac{I_{1}(\beta R)}{I_{0}(\beta R)} e^{i \omega t}
\end{aligned}
$$

where $m=1,2$ are for case I and II, respectively.

The approximate transverse isotropic poroelastic constants for osteon and some necessary geometrical constants are grouped in Table 1 . We select $0.04-0.3 \%$ [1, 33-35] as the strain range to investigate its relationship to the canalicular fluid velocity, pressure, flow rates, and shear stress. A physiological frequency range of 1-20 Hz [27] was selected to study the role of loading frequency. The canalicular radius ranged from 0.1 to $1 \mu \mathrm{m}[38,39]$.

\section{Results}

\section{Role of loading factor: axial strain amplitude $\varepsilon_{z 0}$ and frequency}

As shown in Fig. 3, the FFR amplitudes ( $|Q|$, FFRAs) and FSS amplitudes $\left(\left|\tau_{w}\right|\right.$ FSSAs) of the two cases are proportional to the loading amplitude $\left(\varepsilon_{z 0}\right)$ and frequency $(\omega)$. When one of the two parameters $\left(\varepsilon_{z 0}\right.$ or $\left.\omega\right)$ is fixed, both the FFRA and FSSA have a linear relationship with the other parameter. Both the FFRA and FSSA produced in case II are also larger than those of case I.

\section{Dominating role: the strain rate}

The amplitude of the strain rate, $\dot{\varepsilon}_{z}$, can be defined by the relationship of $\dot{\varepsilon}_{z}=\left|i \omega \varepsilon_{z 0} e^{i \omega t}\right|=\varepsilon_{z 0} \omega[1,18-22,33-35]$ and flowing formulas for each case are obtained according to Eqs. (8), (9):

$$
\left|Q_{m}\right|=\left|\frac{2 \pi}{\omega^{2} \rho l} p_{0 m}^{*}(b)\left[\frac{R}{\beta} \frac{I_{1}(\beta R)}{I_{0}(\beta R)}-\frac{R^{2}}{2}\right]\right| \dot{\varepsilon}_{z} ; \quad m=1,2,
$$

Table 1 Geometrical and material characteristics used in the model [36]

\begin{tabular}{lllllll}
\hline$E_{r}(\mathrm{GPa})$ & $\boldsymbol{\mu}_{\boldsymbol{r}}$ & $\boldsymbol{E}_{\boldsymbol{z}}(\mathrm{GPa})$ & $\boldsymbol{\mu}_{\boldsymbol{z}}$ & $\mathbf{M}(\mathrm{GPa})$ & $\boldsymbol{a}(\boldsymbol{\mu m})$ & $\boldsymbol{b}(\boldsymbol{\mu m})$ \\
\hline 15.9 & 0.328 & 20.3 & 0.25 & 38 & 50 & 150 \\
\hline $\mathbf{a}$ & $\mathbf{a}^{\prime}$ & $\boldsymbol{k}\left(\mathbf{m}^{\mathbf{2}}\right)$ & $\boldsymbol{\mu}(\mathrm{Pas})$ & $\boldsymbol{R}(\mathrm{m})$ & $\boldsymbol{\rho}\left(\mathrm{Kg} \mathrm{m}^{-\mathbf{3}}\right)$ & \\
\hline 0.132 & 0.092 & $10^{-18}$ & $10^{-3}$ & $1-10 \times 10^{-7}$ & 1000 & \\
\hline
\end{tabular}



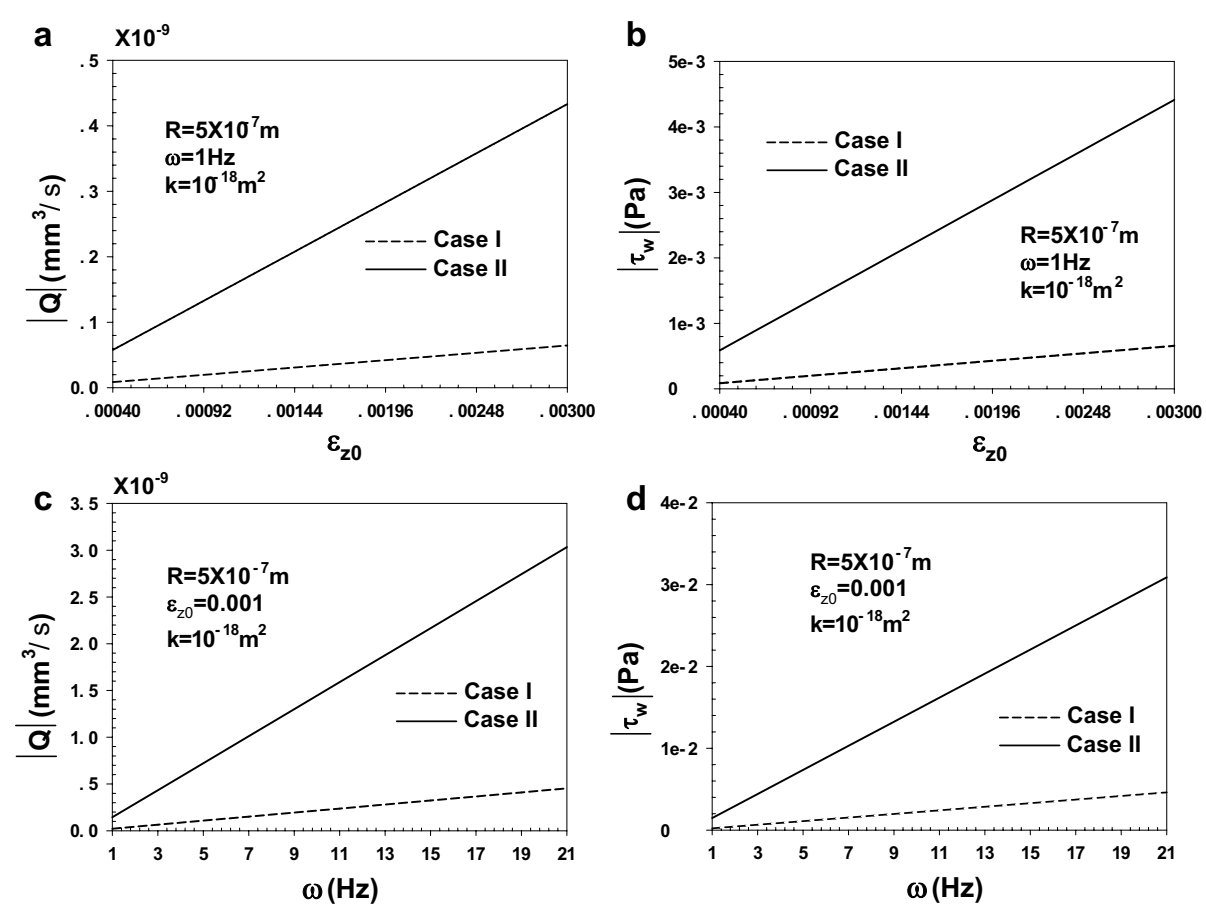

Fig. 3 Fluid flow rate amplitude $(|Q|$, FFRA $)$ and fluid shear stress amplitude $\left(\mid \tau_{w}\right.$, , FSSA $)$ as a function of the loading amplitude $\left(\varepsilon_{z 0}\right)$ and frequency $(\omega)$ at $R=5 \times 10^{-7} \mathrm{~m}$ and $\mathrm{k}=10^{-18} \mathrm{~m}^{2}$. a Fluid flow rate amplitude as a function of the loading amplitude. $\mathbf{b}$ Fluid shear stress amplitude as a function of the loading amplitude. $\mathbf{c}$ Fluid flow rate amplitude as a function of the frequency. $\mathbf{d}$ Fluid shear stress amplitude as a function of the frequency

$$
\left|\tau_{w m}\right|=\left|\frac{\mu \beta}{\omega^{2} \rho l} p_{0 m}^{*}(b) \frac{I_{1}(\beta R)}{I_{0}(\beta R)}\right| \dot{\varepsilon}_{z} ; \quad m=1,2,
$$

where $p_{0 m}^{*}(b)=p_{0 m}(b) / \varepsilon_{z 0}, p_{0 m}^{*}(b)=p_{0 m}(b) / \varepsilon_{z 0}$. As shown in formulas (21)-(22), the FFRAs and FSSAs of the two cases are proportional to the amplitude of strain rate $\left(\dot{\varepsilon}_{z}\right)$.

As shown in Fig. 4, the FFRAs and FSSAs of the two cases change little with the increase in frequency from 1 to $21 \mathrm{~Hz}$ with the strain rate fixed. Both the FFRA and FSSA depend more on the strain rate than on the loading frequency in a physiological loading state. Hence, the key role governing the fluid flow behavior and the FSS is the strain rate.

\section{Time responses of FFR and FSS}

Figure 5 demonstrates the evolution of strain load with time. Based on Fig. 6, the evolutions of FFR and FSS with time are illustrated in Fig. 5. With the cyclic load, the induced FFR and FSS in the canaliculi are also cyclic, as shown in Fig. 6. Both the FFR and FSS in case II are larger (approximately 6.7 times) than those of case I.

\section{Effects of canalicular radius}

Figure 7 illustrates the evolution of FFRA and FSSA with the canalicular radius. As shown in Fig. 7a and c, the FFRAs of both cases have a nonlinear increasing relationship with the canaliculus radius. The FSSAs of the two cases are proportional to the canalicular radius. 

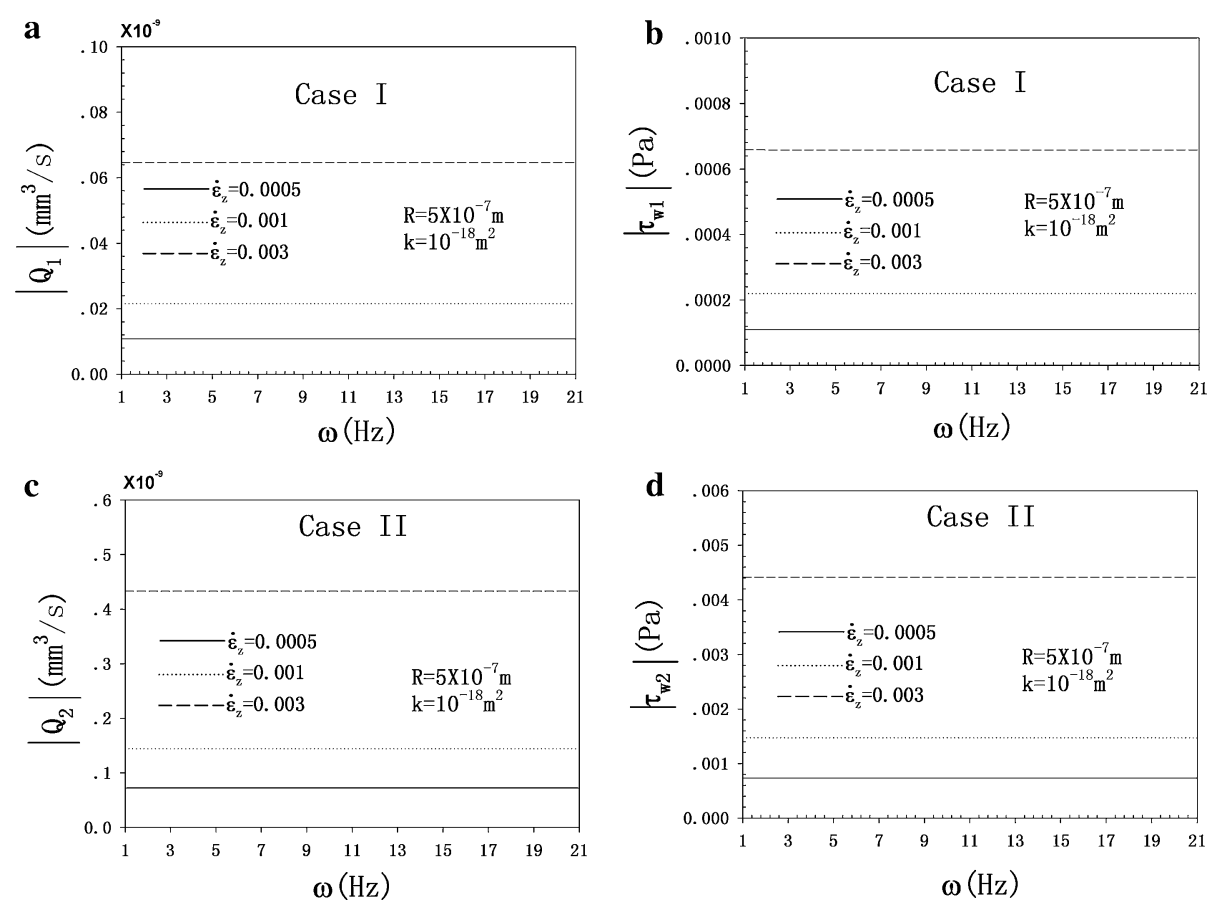

Fig. 4 FFRA and FSSA as a function of the loading frequency at $R=5 \times 10^{-7} \mathrm{~m}, k=10^{-18} \mathrm{~m}^{2}$ and with the strain rate fixed at $\dot{\varepsilon}_{z}=0.0005,0.001$, and $0.003 \mathrm{~s}^{-1}$. a FFRA as a function of the frequency for case I. b FSSA as a function of the frequency for case I. c FFRA as a function of the frequency for case II. d FFSA as a function of the frequency for case II

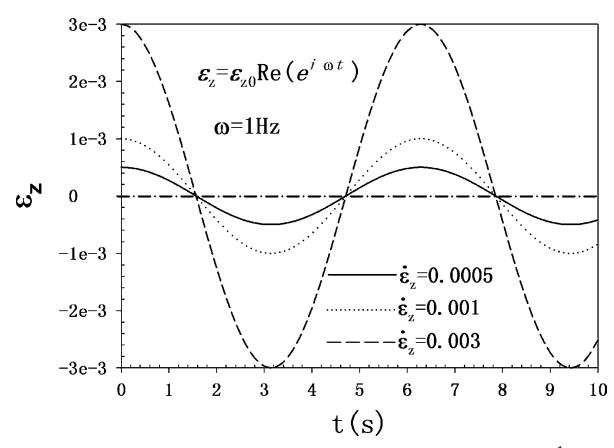

Fig. 5 History of strain loads at $\omega=1 \mathrm{~Hz}$ and $\dot{\varepsilon}_{z}=0.0005,0.001$, and $0.003 \mathrm{~s}^{-1}$, the operator Re () gives the real part of the complex number

\section{Role of permeability}

The role of permeability is examined in Fig. 8 and plotted with the strain loading rate fixed at $\dot{\varepsilon}_{z}=0.001 \mathrm{~s}^{-1}$. According to [19] and [40], this parameter lies between $10^{-23}$ and $10^{-18} \mathrm{~m}^{2}$. We chose $10^{-18} \mathrm{~m}^{2}$ as a reference case to obtain the above results. The FFRAs and FSSAs of both cases experience a monotonic decrease with permeability from $10^{-23}$ to $10^{-18} \mathrm{~m}^{2}$, as shown in Fig. 8 . 

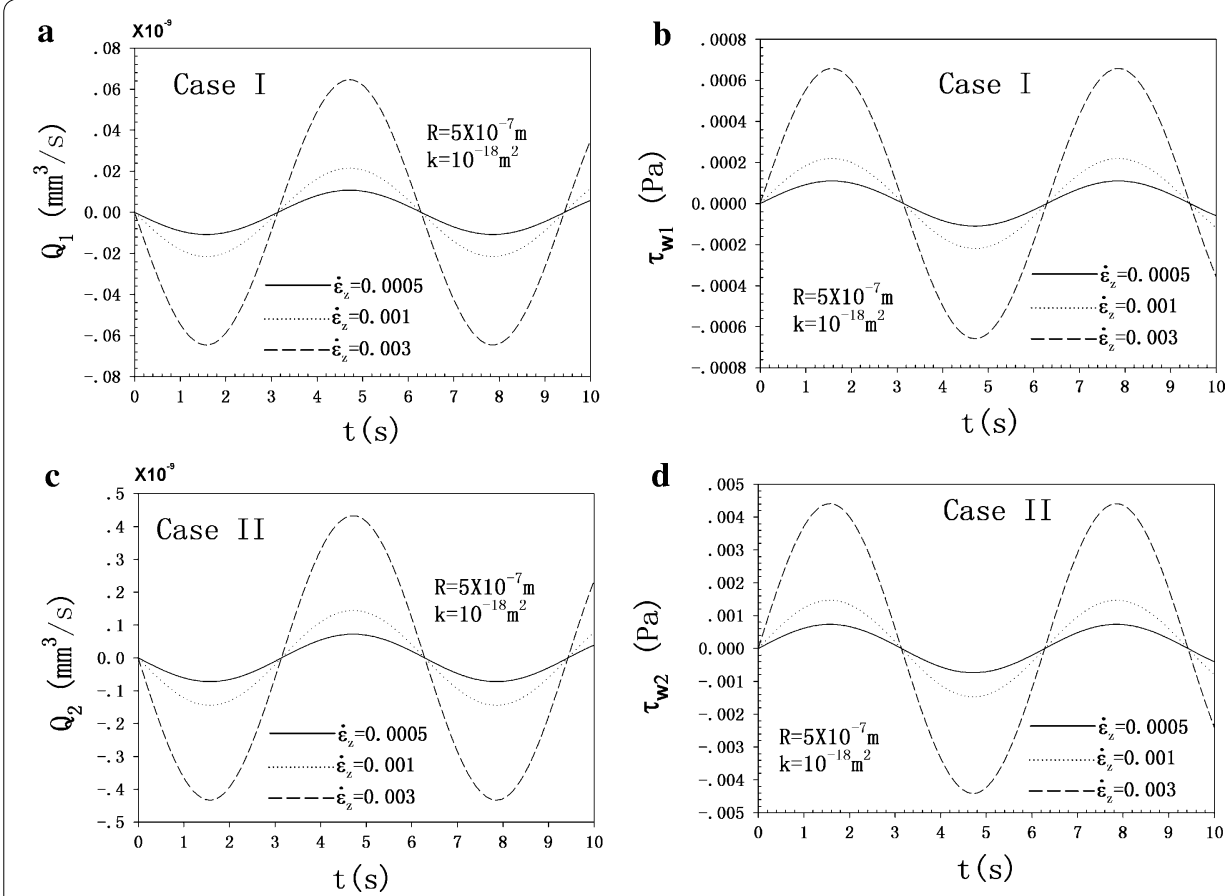

Fig. 6 Time responses of FFR (a Case l; c Case II) and FSS (b Case l; $\mathbf{d}$ Case II) at $R=5 \times 10^{-7} \mathrm{~m}, \mathrm{k}=10^{-18} \mathrm{~m}^{2}$
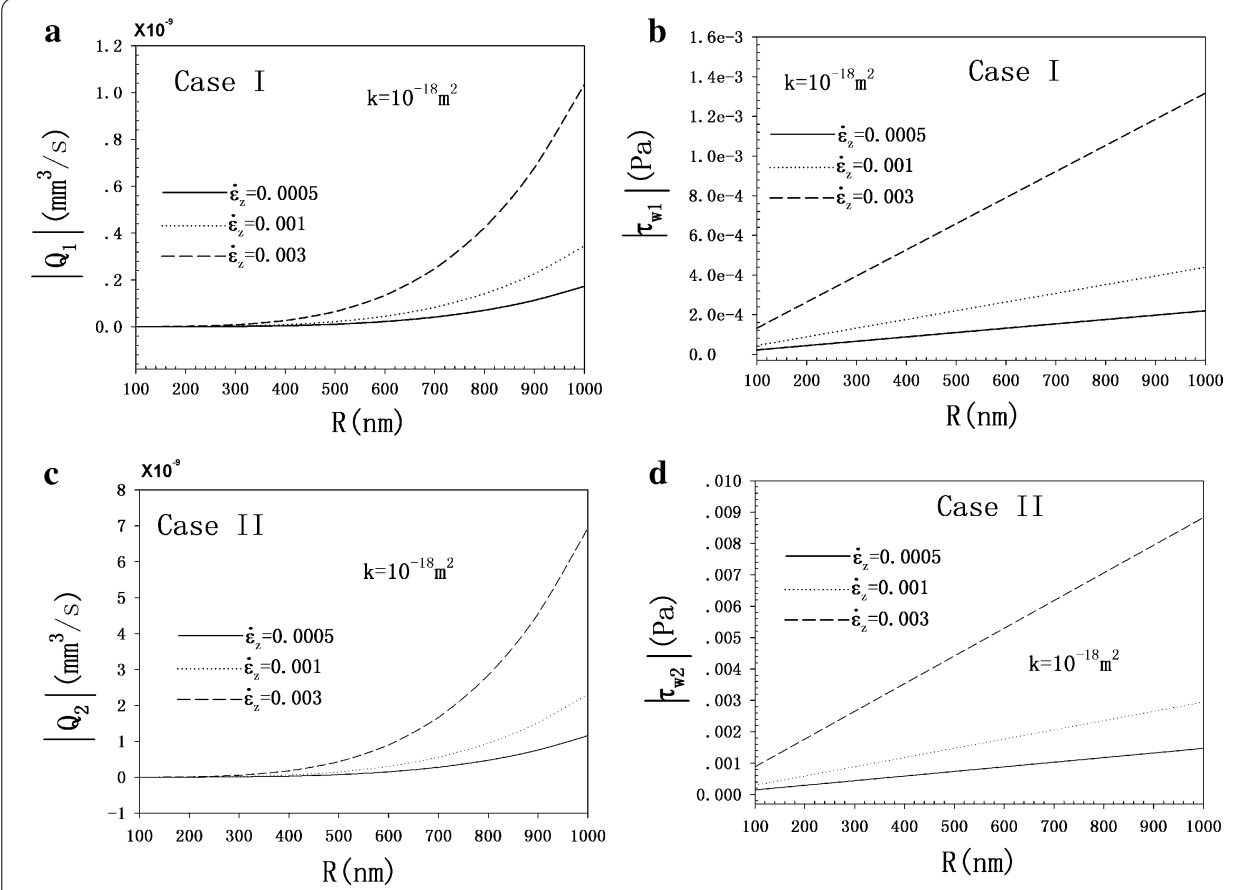

Fig. 7 Evolutions of FFRA $(\mathbf{a}, \mathbf{c})$ and FSSA $(\mathbf{b}, \mathbf{d})$ with canalicular radius at $k=10^{-18} \mathrm{~m}^{2}$

\section{Discussion}

We present a theoretical model to describe the fluid flow and shear stress in the canaliculi of an osteon under axial harmonic loading. The current model links the mechanical 

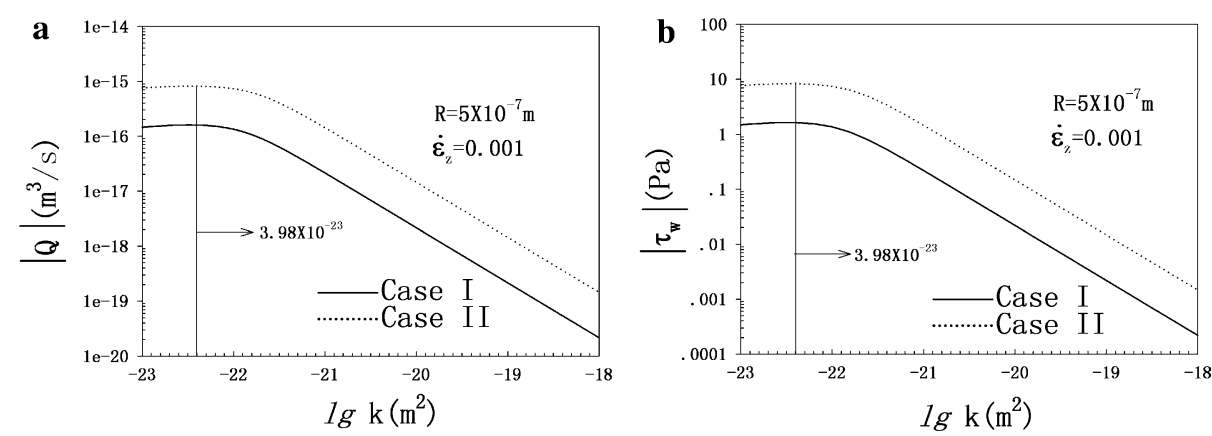

Fig. 8 Effects of permeability with parameter values of $R=1 \times 10^{-7} \mathrm{~m}, \dot{\varepsilon}_{0}=0.001 \mathrm{~s}^{-1}$. Case I (solid line); case II (dotted line). a FFRA as a function of the permeability. $\mathbf{b}$ FSSA as a function of the permeability

loading at the osteon scale to the scale of canalicular fluid pressure, velocity, flow rates, and shear stress, which may have a significant stimulus to bone mechanotransduction.

We have developed the analytical solutions of the FFR and FSS as theoretical basis, and we aim to see the effects of the variation in boundary conditions and material parameters on the FFR and FSS. Loading boundary and the material parameters are the two major groups of factors that influence the FFR and FSS induced in the canaliculi. The linear increase of loading factors-strain amplitude and frequency-leads to the linear increase of FFRA and FSSA (see Fig. 4). However, the strain rate is the real loading factor for governing the canalicular fluid flow behavior (see Fig. 5). Although the actual fluid flow rates have not been measured in vivo, in vivo bone strains have been directly measured in humans (1200-1900 $\mu \varepsilon$ [39]) during vigorous physical activities. The selected strain range of $0.04-0.3 \%$ cannot provide enough FSS $(0.2-6 \mathrm{~Pa},[23,39])$ for the cell to respond. However, some studies [41-43] indicate that a threshold of between 5 and 10\% strain may generate this response. Therefore, the strain amplitude should be increased in the present investigation. A physiological frequency range of $1-20 \mathrm{~Hz}$ [38] is selected to examine its effect on FFR and FSS. The frequency has a substitutable role as the strain amplitude does on the FFR and FSS. This phenomenon could explain why Fritton et al. [44] observed that low-magnitude high-frequency mechanical loading appeared to have the same effect on maintaining bone mass. Live bones experience different physiological loading amplitudes for different frequencies, and loading amplitude has been reported to decrease as the frequency increases [44]. The loading amplitude and frequency are thus coupled in a physiological state. Therefore, the defined parameter, $\varepsilon_{\mathrm{z} 0} \cdot \omega$ (strain rate amplitude), is reasonable and crucial, and it can be considered a representative loading parameter under a physiological state.

Canalicular fluid flow behavior depends not only on loading conditions, but also on geometric characteristics and material parameters. Figure 8 shows that the FFRAs and FSSAs increase as the canalicular radius increases. A large canalicular radius means the cross section where mass flux flows through is large, thereby increasing the FFRA. Permeability can be regarded as the macroscopic indicator of fluid flow at the microscopic level [19]. Published studies on determining the value of $k$ mainly differ in terms of the bone scale. For heterogeneous structures, the local permeability value is related to a particular location on the osteon sample, and several experiments are required to obtain a 
representative or average value [45]. We selected the value $10^{-18} \mathrm{~m}^{2}$ as a reference case for the osteon associated to microscopic lacuno-canalicular.

The boundary conditions of case I allow fluid passage from the inner osteon wall and none across the outer elastic constrained wall. It can be assumed that the environmental liquid around the osteon can automatically produce physiological pressure on the cement surface to balance the pore pressure [33]. This boundary condition suggests that pore pressure is equal to the pressure of physiological liquid around the osteon. In case II, the fluid can freely passage from the inner wall but none across the outer wall, which is almost impossible or unrealistic for the osteon, while, it might be applicable and helpful for geomechanic engineering problems. In this case, the osteon cement surface is supposed to be perfectly rigid without fluid flowing through this surface (impermeable). This displacement boundary is an essential condition to obtain the analytical solutions. This assumption, which has been used in previous studies, may be rough and strong [18-23]. Superficially, case I seems closer to the physiological state than case II. Besides, as shown in Fig. 7, both the FFR and FSS induced in case II are larger (approximately 6.7 times) than those of case I.

In the model of Literature [6], the porous matrix medium is treated as isotropic material, while ours is transversely isotropic (osteon). They describe the fluid annulus surrounding the osteocytic process by a Brinkman equation for a fiber filled medium, while in our model its Navier-Stokes momentum equation. In our model, we disregarded the effects of fluid exchange in the lacuna and fiber matrix and focus on the first physical effects of canalicular fluid transport behavior-shear stress. Though their model seems more accurate, it is complicated and do not linking of cyclic mechanical loading on osteon scale to the fluid flow in the canaliculi scale. There are some unaddressed controversies in the view of morphology and anatomy. Such as theory must be a osteocyte processes fiber lays in the canaliculi and how many fibers each canaliculi contains? These issues have a large extent affecting the modelling method.

Strain-derived interstitial fluid stimuli, such as FSS [6] and fluid pressure gradient [46], serve an important function in bone mechanotransduction. Some other phenomena, such as piezoelectricity $[47,48]$, streaming potential $[36,49,50]$, and chemotransport [51] in the PLC have also been postulated as mechanotransduction mechanisms for positive bone adaptation. Hence, further works, such as calculating these coupled physical effects generalized in the canaliculi and their role in cell response, need to be conducted.

The Hierarchical modeling processes have several limitations: (1) The proposed osteon boundary cases are the idealization of physiological conditions. (2) Every canaliculus is assumed to straightly run across the osteon wall and neglect the effect of lacuna. (3) A homogenized distribution of the canaliculus leads the permeability to be a constant. (4) One-dimensional (only longitudinal velocity component) canalicular fluid flow is considered.

\section{Conclusions}

This hierarchical model firstly provides the analytical estimate of canalicular fluid flows in an axial loaded osteon. At the canaliculus scale, the fluid flow is described by a Stokes equation and its directly stimuli effect-FSS is focused on. This approach will enhance the evaluation of canalicular fluid flow related to bone mechanotransduction, and could 
also potentially account for other geological engineering problems. Despite the limitations of this study, the following conclusions are drawn:

I. The amplitudes of FFR and FSS are proportional to the strain amplitude and frequency. However, the key loading factor governing the FFR and FSS is the strain rate and it can be considered as a representative loading parameter in a physiological state.

II. The larger canalicular radius is, the larger FFRA and FSSA generalized, and the FSSA is proportional to the canalicular radius.

III.At the osteon scale, both the FFRAs and FSSAs experience a monotonic decrease as permeability increases.

IV.Both the FFR and FSS produced in case II (displacement confined osteon outer wall) are larger (approximately 6.7 times) than those of case I (elastic restrained osteon outer wall).

\section{Abbreviations}

FSS: fluid shear stress; FFR: fluid flow rate; PLC: lacunar-canalicular porosity; FFRA: fluid shear stress amplitude; FSSA: fluid flow rate amplitude; FFRAs: fluid shear stress amplitudes; FSSAs: fluid flow rate amplitudes.

\section{Declarations}

\section{Authors' contributions}

XW was responsible for the design, data collection and overall investigation. NW, ZW and WY were responsible for computational modeling and data analysis part. YW and YG were responsible for modifying languages and designing the content. WC was responsible for conducting the content. All authors (1) have made substantial contributions to conception and design, or acquisition of data, or analysis and interpretation of data; (2) have been involved in drafting the manuscript or revising it critically for important intellectual content; and (3) have given final approval of the version to be published. Each author has participated sufficiently in the work to take public responsibility for appropriate portions of the content. All authors read and approved the final manuscript.

\section{Acknowledgements}

This work was supported by the National Natural Science Foundation of China (No. 11302143,11632013), Natural Science Foundation of Shanxi (No. 2014021013), the Scientific and Technological Innovation Projects of Colleges and Universities in Shanxi Province (No. 2014116), and the financial support from the Program for the Outstanding Innovative Teams of Higher Learning Institutions of Shanxi.

Competing interests

The authors declare that they have no competing interests.

\section{About this supplement}

This article has been published as part of BioMedical Engineering OnLine Volume 15 Supplement 2, 2016. Computational and experimental methods for biological research: cardiovascular diseases and beyond. The full contents of the supplement are available online http://biomedical-engineering-online.biomedcentral.com/articles/supplements/ volume-15-supplement-2.

\section{Availability of data and materials}

Data is available from the corresponding author upon reasonable request.

Funding

Publication charges for this article have been funded by the National Natural Science Foundation of China (No. 11302143, 11632013).

Published: 28 December 2016

\section{References}

1. Wu XG, Chen WY, Gao ZP, et al. The effects of haversian fluid pressure and harmonic axial loading on the poroelastic behaviors of a single osteon. Sci China Phys Mech Astron. 2012;55(9):1646-56.

2. Wang L, Ciani C, Doty SB, Fritton SP. Delineating bone's interstitial fluid pathway in vivo. Bone. 2004;34(3):499-509

3. Cowin SC. Bone poroelasticity. J Biomech. 1999;32(3):217-38. 
4. Burger EH, Klein-Nulend J. Mechanotransduction in bone: role of the lacuno-canalicular network. FASEB J. 1999;13(9001):101-12.

5. Tate MLK, Knothe U. An ex vivo model to study transport processes and fluid flow in loaded bone. J. Biomech. 2000;33(2):247-54.

6. Weinbaum S, Cowin SC, Zeng YA. A model for the excitation of osteocytes by mechanical loading-induced bone fluid shear stresses. J Biomech. 1994;27(3):339-60.

7. Anderson EJ, Kaliyamoorthy S, Alexander JID. Nano-microscale models of periosteocytic flow show differences in stresses imparted to cell body and processes. Ann Biomed Eng. 2005;33(1):52-62.

8. Ahn A, Grodzinsky A. Relevance of collagen piezoelectricity to Wolff's law: a critical review. Med Eng Phys. 2009;31(7):733-41.

9. Brown TD. Techniques for mechanical stimulation of cells in vitro: a review. J Biomech. 2000;33(1):3-14.

10. Salzstein RA, Pollack SR. Electromechanical potentials in cortical bone-I. A continuum approach. J Biomech. 1987;20(3):261-70.

11. Cowin SC, Weinbaum S, Zeng Y. A case for the bone canaliculi as the anatomical site of strain generated potentials. J Biomech. 1995;28(11):1281-97.

12. Piekarski K, Munro M. Transport mechanism operating between blood supply and osteocytes in long bones. Nature. 1995;269(5623):80-2.

13. Pollack S, Petrov N, Salzstein R. An anatomical model for streaming potentials in osteons. J Biomech. 1984;17(8):627-36.

14. Petrov N, Pollack S, Blagoeva R. A discrete model for streaming potentials in a single osteon. J Biomech. 1989;22(6-7):517-21.

15. Kufahl RH, Saha S. A theoretical model for stress-generated fluid flow in the canaliculi-lacunae network in bone tissue. J Biomech. 1990;23(2):171-80.

16. Zeng Y, Cowin SC, Weinbaum S. A fiber matrix model for fluid flow and streaming potentials in the canaliculi of an osteon. Ann Biomed Eng. 1994;22(3):280-92.

17. Zhang D, Weinbaum S, Cowin SC. On the calculation of bone pore water pressure due to mechanical loading. Int J Solids Struct. 1998;35(34-35):4981-97.

18. Rémond A, Naili S. Transverse isotropic poroelastic osteon model under cyclic loading. Mech Res Commun. 2005;32(6):645-51.

19. Rémond A, Naili S, Lemaire T. Interstitial fluid flow in the osteon with spatial gradients of mechanical properties: a finite element study. Biomech Mod Mechanobiol. 2008;7(6):487-95.

20. Nguyen VH, Lemaire T, Naili S. Numerical study of deformation-induced fluid flows in periodic osteonal matrix under harmonic axial loading. C R Mecanique. 2009;337(5):268-76.

21. Nguyen VH, Lemaire T, Naili S. Anisotropic poroelastic hollow cylinders with damaged periphery under harmonically axial loadings: relevance to bone osteons. Multidisci Mod Mater Struct. 2009:5(3):205-22.

22. Nguyen VH, Lemaire T, Naili S. Poroelastic behaviour of cortical bone under harmonic axial loading: A finite element study at the osteonal scale. Med Eng Phys. 2010;32(4):384-90.

23. Nguyen VH, Lemaire T, Naili S. Influence of interstitial bone microcracks on strain-induced fluid flow. Biomech Mod Mechanobiol. 2011;10(6):963-72.

24. Tate MLK. Whither flows the fluid in bone? An osteocyte's perspective. J Biomech. 2003;36(10):1409-24.

25. Srinivasan S, Gross TS. Canalicular fluid flow induced by bending of a long bone. Med Eng Phys. 2000;22(2):127-33.

26. Fritton SP, Weinbaum S. Fluid and solute transport in bone: flow induced mechanotransduction. Annu Rev Fluid Mech. 2009:41:347-74.

27. Cowin SC. Bone poroelasticity. In: Cowin S, editor. Bone mechanics handbook. 2nd ed. Boca Raton: CRC; 2001. p. $1-31$.

28. Lemaire T, Naili S, Rémond A. Study of the influence of fibrous pericellular matrix in the cortical interstitial fluid movement. J Biomech. 2008;130(1):1-11.

29. Lemaire T, Kaiser J, Naili S, Sansalone V. Modeling of the transport in electrically charged porous media including ionic exchanges. Mech Res Commun. 2010;37(5):495-9.

30. Lemaire T, Sansalonem V, Nailim S. Multiphysical modelling of fluid transport through osteo-articular media. AABC. 2010;82(1):127-44.

31. Lemaire T, Capiez-Lernout E, Kaiser J, et al. A multiscale theoretical investigation of electric measurements in living bone piezoelectricity and electrokinetics. B Math Biol. 2011;73(11):2649-77.

32. Sansalone V, Kaiser J, Naili S, et al. Interstitial fluid flow within bone canaliculi and electro-chemo-mechanical features of the canalicular milieu. Biomech Mod Mechanobiol. 2013;12(3):533-53.

33. Wu XG, Chen WY. A hollow osteon model for examining its poroelastic behaviors: mathematically modeling an osteon with different boundary cases. Eur J Mech Solid. 2013:40:34-49.

34. Wu XG, Chen WY. Poroelastic behaviors of the osteon: a comparison of two theoretical osteon models. Acta Mech Sin. 2013;29(4):612-21.

35. Wu XG, Chen WY, Wang DX. Mathematical osteon model for examining poroelastic behaviors. Appl Math Mech. 2013;34(4):405-16.

36. Wu XG, Yu WL, Cen HP, et al. Hierarchical model for strain generalized streaming potential induced by the canalicular fluid flow of an osteon. Acta Mech Sinica. 2015;31(1):112-21.

37. You L, Cowin SC, Schaffler MB, et al. A model for strain amplification in the actin cytoskeleton of osteocytes due to fluid drag on pericellular matrix. J Biomech. 2001;34(11):1375-86.

38. Boyde A. Scanning electron microscope studies of bone. In: Bourne GH, editor. Biochem Physiol. New York: Bone; 1972. p. 259-310.

39. Cooper RR, Milgram JW, Robinson RA. Morphology of the osteon: an electron microscopic study. J Bone Joint Surg. 1966;48(7):1239-71.

40. Beno T, Yoon YJ, Cowin SC. Estimation of bone permeability using accurate microstructural measurements. J Biomech. 2006;39(13):2378-87. 
41. Buckley MJ, Banes AJ, Levin LG. Osteoblasts increase their rate of division and align in response to cyclic, mechanical tension in vitro. Bone Miner. 1988;4(3):225-36.

42. Brand RA, Stanford CM, Nicolella DP. Primary adult human bone cells do not respond to tissue (continuum) level strains. J Orthop Sci. 2001;6(3):295-301.

43. Harter LV, Hruska KA, Duncan RL. Human osteoblast like cells respond to mechanical strain with increased bone matrix protein production independent of hormonal regulation. Endocrinology. 1995;136(2):528-35.

44. Fritton SP, McLeod KJ, Rubin CT. Cross-species spectral similarity in the strain history of bone. Transactions of 42nd annual meeting, orthopediatric research society.1996. p. 19-22.

45. Malachanne E, Dureisseix D, Canadas P. Experimental and numerical identification of cortical bone permeability. J Biomech. 2008:41(3):721-5.

46. Qin YX, Kaplan T, Saldanha A, et al. Fluid pressure gradients, arising from oscillations in intramedullary pressure, is correlated with the formation of bone and inhibition of intracortical porosity. J Biomech. 2003;36(10):1427-37.

47. Basset C, Becker R. Generation of electric potentials by bone in response to mechanical stress. Science. 1962;137(3535):1063-4.

48. Yasuda I. Piezoelectricity of living bone. J Kyoto Pref Univ Med. 1964;53:325.

49. Otter M, Goheen S, Williams WS. Streaming potentials in chemically modified bone. J Orthop Surg Res. 1988;6(3):346-59.

50. Gross D, Williams WS. Streaming potential and the electromechanical response of physiologically moist Bone. J Biomech. 1982;15(4):277-95.

51. Munro PA, Dunnill P, Lilly MD. Nonporous magnetic materials as enzyme supports: studies with immobilized chymotrypsin. Biotechnol Bioeng. 1977;19(1):101-24.

Submit your next manuscript to BioMed Central and we will help you at every step:

- We accept pre-submission inquiries

- Our selector tool helps you to find the most relevant journal

- We provide round the clock customer support

- Convenient online submission

- Thorough peer review

- Inclusion in PubMed and all major indexing services

- Maximum visibility for your research

Submit your manuscript at www.biomedcentral.com/submit 